\title{
Advances in Ablative Composites of Carbon based Materials: A Review
}

\author{
Colonel Vijay Kumara, Balasubramanian Kandasubramanian ${ }^{\mathrm{b}, *}$ \\ andian Army, Government of India, India \\ ${ }^{b}$ Department of Metallurgical and Materials Engineering, Defence Institute of Advanced \\ Technology (DU), Ministry of Defence, Girinagar, Pune-411025, India \\ * Corresponding Author E-mail: meetkbs@gmail.com
}

Table S1. Ablation properties of $\mathrm{CF}$ reinforced composites with different matrices (G/B: carbon/phenolic green composite; IC/C: carbonized G/B composite; IC/C+1IM: IC/C composite impregnated once with $\mathrm{FA}$; IC/C+CVI: IC/C composite with a $\mathrm{CVI}$ process, without any impregnation process). Reprinted with permission from ref ${ }^{65}$. Copyright 2001 Elsevier. 


\begin{tabular}{|c|c|c|c|c|}
\hline Features & G/B & IC/C & $\mathrm{IC} / \mathrm{C}+2 \mathrm{IM}$ & $\mathrm{IC} / \mathrm{C}+\mathrm{CVI}$ \\
\hline Thickness (mm) & 6.615 & 7.160 & 7.161 & 7.218 \\
\hline Density $\left(\mathrm{g} / \mathrm{cm}^{3}\right)$ & 1.478 & 1.389 & 1.524 & 1.440 \\
\hline Burn-through time (s) & 69.187 & 91.368 & 66.983 & 80.0 \\
\hline Mass loss after test & -25.014 & -12.887 & -15.455 & -9.672 \\
\hline \multicolumn{5}{|l|}{ Erosion rate } \\
\hline $\mathrm{mm} / \mathrm{s}$ & 0.0952 & 0.0791 & 0.1094 & 0.0903 \\
\hline $\mathrm{g} / \mathrm{s}$ & 0.3615 & 0.1413 & 0.2313 & 0.1205 \\
\hline Observed delamination & No & No & No & No \\
\hline \multicolumn{5}{|c|}{ Thermally affected appearance } \\
\hline Front & $\begin{array}{l}\text { Typical } \\
\text { ablation }\end{array}$ & Typical ablation & Typical ablation & $\begin{array}{l}\text { Typical } \\
\text { ablation }\end{array}$ \\
\hline Back & Larger area & Smaller area & Medium area & Smaller area \\
\hline Relative eroded gradient & Lower & Lower & Higher & Lower \\
\hline
\end{tabular}

Table S2. Test data for isothermal oxidation of $\mathrm{C} / \mathrm{C}$ composites with toughened coatings (toughened with $\mathrm{SiC}$ whisker). Reprinted with permission from ref ${ }^{256}$. Copyright 2018 Elsevier. 


\begin{tabular}{|c|c|c|c|c|c|c|}
\hline $\begin{array}{c}\text { Toughening- } \\
\text { Material }\end{array}$ & $\begin{array}{l}\text { Coating- } \\
\text { material }\end{array}$ & $\begin{array}{c}\text { Fabrication } \\
\text { methods }\end{array}$ & $\begin{array}{c}\text { Time Pd } \\
\text { (h) }\end{array}$ & $\begin{array}{c}\text { Temperatures } \\
\left({ }^{\circ} \mathrm{C}\right)\end{array}$ & $\begin{array}{c}\text { Mass loss } \\
\text { (\%) }\end{array}$ & Reference \\
\hline- & $\mathrm{SiC}$ & Slurry + PC & 48 & 1500 & 5.00 & 247 \\
\hline $5 \mathrm{wt} / \% \mathrm{SiCw}$ & $\mathrm{SiC}$ & Slurry + PC & 48 & 1500 & 2.80 & 247 \\
\hline $10 \mathrm{wt} . \% \mathrm{SiCw}$ & $\mathrm{SiC}$ & Slurry + PC & 48 & 1500 & 1.50 & 247 \\
\hline 15 wt. $\% \mathrm{SiCw}$ & $\mathrm{SiC}$ & Slurry + PC & 48 & 1500 & 2.50 & 247 \\
\hline 20 wt. $\% \mathrm{SiCw}$ & $\mathrm{SiC}$ & Slurry + PC & 48 & 1500 & 5.50 & 247 \\
\hline- & MoSi2-SiC-Si & Slurry + PC & 200 & 1500 & 2.31 & 248 \\
\hline 10 wt. $\%$ SiCw & $\mathrm{MoSi} 2-\mathrm{SiC}-\mathrm{Si}$ & Slurry + PC & 200 & 1500 & 0.33 & 248 \\
\hline 15 wt. $\%$ SiCw & MoSi2-SiC-Si & Slurry + PC & 50 & 1400 & 0.74 & 249 \\
\hline 15 wt. $\%$ Sicx & Mosi2-SiC-Si & Slurry + PC & 50 & 1500 & 0.67 & 249 \\
\hline- & $\mathrm{SiC}-\mathrm{CrSi}{ }_{2}$ & Slurry + PC & 50 & 1500 & 2.43 & 250 \\
\hline 15 wt. $\% \mathrm{SiCw}$ & $\mathrm{SiC}-\mathrm{CrSi}{ }_{2}$ & Slurry + PC & 50 & 1500 & 0.68 & 250 \\
\hline $\mathrm{SiCW}$ & $\mathrm{MoSi}_{2}-\mathrm{SiC}$ & Slurry & 2.67 & 1500 & 0.08 & 251 \\
\hline $\mathrm{SiCw}-\mathrm{SiCu}$ & $\begin{array}{l}\mathrm{TaSi}_{2}-\mathrm{MoSi}_{2}- \\
\mathrm{SiC} / \mathrm{MoSi}_{2}-\mathrm{SiC}\end{array}$ & Slurry & 2.67 & 1500 & 0.02 & 251 \\
\hline
\end{tabular}

Table S3. Single fiber tensile test results for reinforced CF \& GF recovered via pyrolysis (r: recycled; v: virgin). Reprinted with permission from ref ${ }^{342}$. Copyright 2015 Elsevier.

\begin{tabular}{|l|l|l|l|l|}
\hline \multicolumn{1}{|c|}{ Process } & \multicolumn{1}{|c|}{ Fiber type } & \multicolumn{3}{c|}{ Single fiber tensile strength } \\
\hline Fluidised bed pyrolysis & Owens Corning & v & $10 \mathrm{~mm}$ & $2.61 \mathrm{GPa}$ \\
& GF roving RO7E & & & \\
& for SMC & & & \\
\hline
\end{tabular}




\begin{tabular}{|c|c|c|c|c|c|}
\hline & & $\mathrm{r}$ & $10 \mathrm{~mm}$ & \multirow{2}{*}{\multicolumn{2}{|c|}{$\begin{array}{l}-52 \% \text { at } 450{ }^{\circ} \mathrm{C} \\
-64 \% \text { at } 550{ }^{\circ} \mathrm{C}\end{array}$}} \\
\hline & & & & & \\
\hline \multirow[t]{4}{*}{ Comparison between } & Grafil 34-700 & $\mathrm{v}$ & & \multicolumn{2}{|c|}{$4.09 \mathrm{GPa}$} \\
\hline & & $\begin{array}{l}\text { Fluidised } \\
\text { bed }\end{array}$ & & \multicolumn{2}{|l|}{$-25 \%$} \\
\hline & & Microwave & & \multicolumn{2}{|l|}{$-20 \%$} \\
\hline & & $\mathrm{scPrOH}$ & & \multicolumn{2}{|l|}{$-5 \%$} \\
\hline \multirow[t]{2}{*}{$\begin{array}{l}\text { Refiber optimised } \\
\text { pyrolysis }\end{array}$} & $\begin{array}{l}\text { Toho-Tenax } \\
\text { HTA }\end{array}$ & $\mathrm{v}$ & & \multicolumn{2}{|c|}{$3.712 \mathrm{GPa}$} \\
\hline & & $\mathrm{r}$ & & \multicolumn{2}{|l|}{$-4 \%$} \\
\hline \multirow[t]{2}{*}{ ELGCF pyrolysis } & $\begin{array}{l}\text { TR505 CF from } \\
\text { Pyrofil }\end{array}$ & $\mathrm{v}$ & $25 \mathrm{~mm}$ & \multicolumn{2}{|c|}{$3.19 \mathrm{GPa}$} \\
\hline & & $\mathrm{r}$ & $25 \mathrm{~mm}$ & \multicolumn{2}{|c|}{$\begin{array}{l}-10 \% \text { at } 500{ }^{\circ} \mathrm{C}- \\
10 \mathrm{~min} \text { in air }\end{array}$} \\
\hline \multirow[t]{8}{*}{ ELG pyrolysis } & Hexcel AS4 & $\mathrm{v}$ & $10 \mathrm{~mm}$ & \multicolumn{2}{|c|}{$4.897 \mathrm{GPa}$} \\
\hline & & & $20 \mathrm{~mm}$ & \multicolumn{2}{|c|}{$4.551 \mathrm{GPa}$} \\
\hline & & r-A & $10 \mathrm{~mm}$ & $-83 \%$ & \multirow{6}{*}{ Decreasing } \\
\hline & & & $20 \mathrm{~mm}$ & $-85 \%$ & \\
\hline & & $\mathrm{r}-\mathrm{B}$ & $10 \mathrm{~mm}$ & $-75 \%$ & \\
\hline & & & $20 \mathrm{~mm}$ & $-81 \%$ & \\
\hline & & $\mathrm{r}-\mathrm{C}$ & $10 \mathrm{~mm}$ & $-67 \%$ & \\
\hline & & & $20 \mathrm{~mm}$ & $-76 \%$ & \\
\hline
\end{tabular}




\begin{tabular}{|c|c|c|c|c|c|}
\hline & & r-D & $10 \mathrm{~mm}$ & $-2 \%$ & \\
\hline & & & $20 \mathrm{~mm}$ & $-4 \%$ & \\
\hline ENEA pyrolysis process & Torayca T800S & $\mathrm{v}$ & & $5.9 \mathrm{GP}$ & \\
\hline & & $\mathrm{r}$ & $10 \mathrm{~mm}$ & $-17 \%$ & \\
\hline & & & $20 \mathrm{~mm}$ & $-24 \%$ & \\
\hline & & r 450 & $10 \mathrm{~mm}$ & $-17 \%$ & $\begin{array}{l}\text { Post-treated } \\
\text { at } 450{ }^{\circ} \mathrm{C}\end{array}$ \\
\hline & & & $20 \mathrm{~mm}$ & $-36 \%$ & \\
\hline & & r 600 & $10 \mathrm{~mm}$ & $-69 \%$ & $\begin{array}{l}\text { Post-treated } \\
\text { at } 600{ }^{\circ} \mathrm{C}\end{array}$ \\
\hline & & & $20 \mathrm{~mm}$ & $-82 \%$ & \\
\hline & & $r$ acid & $10 \mathrm{~mm}$ & $-24 \%$ & $\begin{array}{l}\text { Post-treated } \\
\text { in nitric acid }\end{array}$ \\
\hline & & & $20 \mathrm{~mm}$ & $-44 \%$ & \\
\hline $\begin{array}{l}\text { Materials Innovation } \\
\text { Technologies pyrolysis }\end{array}$ & $\begin{array}{l}\text { CFs } \\
\text { (diverse } \\
\text { feedstock) }\end{array}$ & $\begin{array}{l}\text { Loss } 0 \\
\text { to virg }\end{array}$ & $\begin{array}{l}\text { ompared } \\
\text { S fibers }\end{array}$ & m Tora & \\
\hline
\end{tabular}

Table S4. Energy intensity with respect to resin and fiber production. Reprinted with permission from ref ${ }^{342}$. Copyright 2015 Elsevier. 


\begin{tabular}{|c|c|}
\hline Material & Energy intensity for production $(\mathrm{MJ} / \mathrm{kg})$ \\
\hline \multicolumn{2}{|l|}{ Resins } \\
\hline Polyester & 63-78 \\
\hline Epoxy & $76-80$ \\
\hline PP & $72-112$ \\
\hline \multicolumn{2}{|l|}{ Fibers } \\
\hline GF & $13-32$ \\
\hline $\mathrm{CF}$ & $183-286$ \\
\hline Flax fibre & 6.5 \\
\hline \multicolumn{2}{|l|}{ Metals } \\
\hline Aluminium & $196-257$ \\
\hline Stainless steel & $110-210$ \\
\hline Steel & $30-60$ \\
\hline
\end{tabular}

